Open Access

\title{
Optimal esophageal balloon volume for accurate estimation of pleural pressure at end-expiration and end-inspiration: an in vitro bench experiment
}

Yan-Lin Yang ${ }^{1,2 \dagger}$, Xuan He ${ }^{1 \dagger}$, Xiu-Mei Sun ${ }^{1}$, Han Chen ${ }^{1,3}$, Zhong-Hua Shi', Ming Xu' ${ }^{1}$ Guang-Qiang Chen ${ }^{1}$ and Jian-Xin Zhou ${ }^{1 *}$

\section{* Correspondence:}

zhoujx.cn@icloud.com

${ }^{\dagger}$ Equal contributors

'Department of Critical Care Medicine, Beijing Tiantan Hospital, Capital Medical University, No 6, Tiantan Xili, Dongcheng District, Beijing 100050, China

Full list of author information is available at the end of the article

\begin{abstract}
Background: Esophageal pressure, used as a surrogate for pleural pressure, is commonly measured by air-filled balloon, and the accuracy of measurement depends on the proper balloon volume. It has been found that larger filling volume is required at higher surrounding pressure. In the present study, we determined the balloon pressure-volume relationship in a bench model simulating the pleural cavity during controlled ventilation. The aim was to confirm whether an optimal balloon volume range existed that could provide accurate measurement at both end-expiration and end-inspiration.

Methods: We investigated three esophageal balloons with different dimensions and materials: Cooper, SmartCath-G, and Microtek catheters. The balloon was introduced into a glass chamber simulating the pleural cavity and volume-controlled ventilation was initiated. The ventilator was set to obtain respective chamber pressures of 5 and $20 \mathrm{cmH}_{2} \mathrm{O}$ during end-expiratory and end-inspiratory occlusion. Balloon was progressively inflated, and balloon pressure and chamber pressure were measured. Balloon transmural pressure was defined as the difference between balloon and chamber pressure. The balloon pressure-volume curve was fitted by sigmoid regression, and the minimal and maximal balloon volume accurately reflecting the surrounding pressure was estimated using the lower and upper inflection point of the fitted sigmoid curve. Balloon volumes at end-expiratory and end-inspiratory occlusion were explored, and the balloon volume range that provided accurate measurement at both phases was defined as the optimal filling volume.

Results: Sigmoid regression of the balloon pressure-volume curve was justified by the dimensionless variable fitting and residual distribution analysis. All balloon transmural pressures were within $\pm 1.0 \mathrm{cmH}_{2} \mathrm{O}$ at the minimal and maximal balloon volumes. The minimal and maximal balloon volumes during end-inspiratory occlusion were significantly larger than those during end-expiratory occlusion, except for the minimal volume in Cooper catheter. Mean ( \pm standard deviation) of optimal filling volume both suitable for end-expiratory and end-inspiratory measurement ranged $0.7 \pm 0.0$ to $1.7 \pm$ $0.2 \mathrm{ml}$ in Cooper, $1.9 \pm 0.2$ to $3.6 \pm 0.3 \mathrm{ml}$ in SmartCath-G, and $2.2 \pm 0.2$ to $4.6 \pm 0.1 \mathrm{ml}$ in Microtek catheter.

(Continued on next page)
\end{abstract}


(Continued from previous page)

Conclusions: In each of the tested balloon, an optimal filling volume range was found that provided accurate measurement during both end-expiratory and end-inspiratory occlusion.

Keywords: Esophageal balloon, Balloon volume, Sigmoid regression, Measurement, Bench experiment

\section{Background}

Esophageal pressure has been used as a surrogate for pleural pressure in the monitoring of respiratory mechanics for many years [1, 2]. Previously, esophageal pressure measurement was mainly used to assess respiratory muscle effort and the work of breathing during spontaneous or assisted ventilation, particularly in patients with difficult weaning $[3,4]$. More recently, this technique has been extended to guide lungprotective ventilation in patients with acute respiratory distress syndrome [5-8].

Esophageal pressure is commonly measured by catheter with air-filled balloon, and the accuracy of measurement depends on the proper filling of the balloon $[1,2]$. Studies have demonstrated that overfilling of the balloon might overestimate esophageal pressure; in contrast, underfilling might lead to underestimation [9-16]. Theoretically, the optimal balloon volume is the range with minimal discrepancy between the pressures measured inside and outside of the balloon, namely the minimal balloon transmural pressure $\left(P_{\mathrm{TM}}\right)$. It has been found that under bench conditions, greater balloon volume is required for pressure transmission at higher surrounding pressure [15]. Therefore, during passive ventilation, the optimal balloon volume obtained during the end-expiratory phase (low pressure) might not be simultaneously suitable for the measurement during the end-inspiratory phase (high pressure), especially in patients with high driving pressure. This possibility warrants further investigation.

Previous studies have shown a nonlinear sigmoid shape of the balloon pressurevolume curve under in vitro and in vivo conditions [9, 11-17]; however, no study has attempted this type of regression analysis. We speculated that the sigmoid fitting of balloon pressure-volume curve might provide an additional method to determine the optimal balloon volume. In the present study, we selected three commercially available esophageal catheters with different balloon dimensions and materials. The balloon pressure and volume data were fitted using a sigmoid regression equation, and the optimal balloon volume was determined under atmospheric pressure and in a dynamic bench model simulating the pleural cavity during volume-controlled ventilation. The overall goodness-of-fit of the sigmoid regression and the accuracy of the balloon volume estimation were assessed. $P_{\mathrm{TM}}$ was determined, and an acceptable accuracy of measurement was defined as a $P_{\mathrm{TM}}$ within $\pm 1 \mathrm{cmH}_{2} \mathrm{O}$ [15]. We aimed to confirm whether an optimal balloon volume range existed that could provide accurate measurement at both end-expiration and end-inspiration.

\section{Methods}

\section{Esophageal balloon catheters}

Three commercially available esophageal balloon catheters with different balloon dimensions and materials were tested: (1) Cooper catheter (LOT 177405, Cooper Surgical, USA) 
of $5 \mathrm{Fr}$ in diameter and $85 \mathrm{~cm}$ in length, enclosed with a polyethylene balloon, and a manufacturer-recommended inflation volume of 1-2 ml; (2) SmartCath-G catheter (LOT 7003300, CareFusion Co., USA) of $16 \mathrm{Fr}$ in diameter and $114 \mathrm{~cm}$ in length, with a polyethylene balloon and a manufacturer-recommended inflation volume of 0.5-2.5 ml; and (3) Microtek catheter (LOT 20110815, Microtek Medical B.V., Netherlands) of 8 Fr in diameter and $100 \mathrm{~cm}$ in length, with a latex balloon and no manufacturer-recommended inflation volume.

For each type of catheter, six samples were randomly selected. Before the experiment, each balloon was tested for leak by inflating under water. The length and diameter of the balloon were measured in one randomly selected catheter of each type using a Vernier caliper gauge (CD67-S, PM/PS, Mitutoyo Measuring Instruments (Shanghai) Co., Ltd., Shanghai, China). The geometric volume of the balloon was calculated based on the length and diameter of the balloon and using the capsule body as an approximation. Because the feeding tube passes through the inner space of the balloon in the SmartCath-G catheter, this volume was deducted when calculating the balloon geometric volume for this type of catheter.

\section{Pressure measurements}

All pressure measurements were performed using pressure transducers (KT 100D-2, Kleis TEK, Italy, range: $\pm 100 \mathrm{cmH}_{2} \mathrm{O}$ ) connected to an ICU-Lab Pressure Box (ICU Lab, KleisTEK Engineering, Bari, Italy) by $80-\mathrm{cm}$ rigid tube lines. Pressure transducers were calibrated using a water column. Signals were displayed continuously and saved (ICU-Lab 2.5 Software Package, ICU Lab, KleisTEK Engineering, Bari, Italy) on a laptop computer for further analysis, at a sample rate of $200 \mathrm{~Hz}$.

\section{Balloon volume manipulation at atmospheric pressure}

The balloon pressure-volume relationship was determined at atmospheric pressure using a previously reported method with some modifications $[12,13,16]$. The balloon lumen of the catheter was connected with a three-way stopcock for volume manipulation of the balloon, and the other two ways were connected to a 1-ml gas-tight syringe (LOT JM00B25, Runze fluid control equipment, C.O., Ltd., Nanjing, China) and a pressure transducer. The balloon was first inflated up to the geometric volume to unfold the balloon's wall. The balloon was then deflated by generating a negative pressure of approximately $-10 \mathrm{cmH}_{2} \mathrm{O}$, and the stopcock was closed. This procedure standardized the residual volume in the balloon, and we arbitrarily defined the balloon volume in this situation as zero inflating volume $[12,13,16]$. Thereafter, the balloon was progressively inflated in $0.5-\mathrm{ml}$ increments. After each volume inflation, balloon pressure was measured after a 3-min equilibration period. Balloon inflation was stopped until the balloon pressure exceeded $+10 \mathrm{cmH}_{2} \mathrm{O}$. Because the experiment under static conditions was performed at atmospheric pressure, $P_{\mathrm{TM}}$ was equal to the balloon pressure.

\section{Balloon volume manipulation under simulated passive ventilation}

A model was constructed to simulate the pleural cavity and the lung (Fig. 1). The model consisted of a 5000-ml glass chamber (Shuniu Glass Instrument Co., Ltd., Sichuan, China) with three openings, a 1000-ml rubber test lung (Puritan-Bennett 


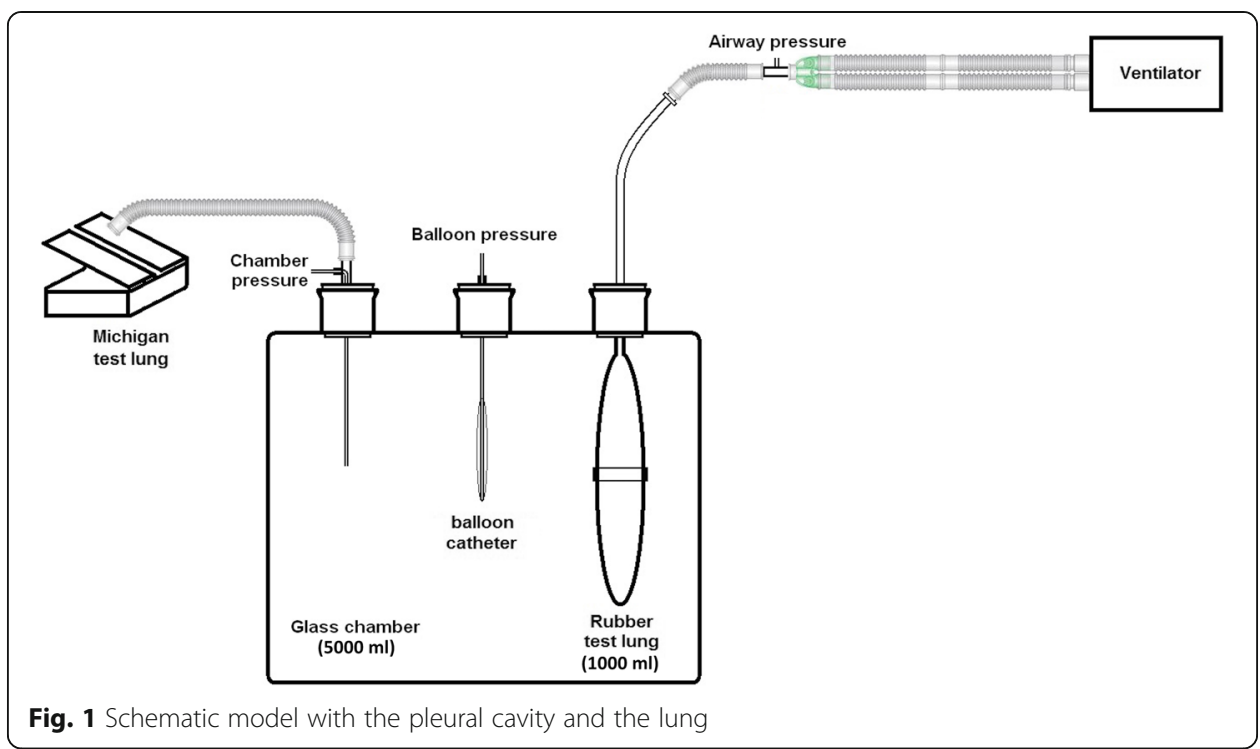

Corporation, CA, USA), and a Michigan test lung (Dual Adult Training Test Lung, Model 5600i, Part Number 15090, Michigan Instruments, Grand Rapids, MI, USA). The rubber test lung was sealed in the glass chamber through one opening to simulate the lung and was connected to a Servo-I ventilator (Maquet Critical Care, Solna, Sweden) using an 8.0 ID endotracheal tube (LOT 1892776, Portex Tracheal Tube, Smiths Medical International Ltd., Keene, USA). The glass chamber was connected to the Michigan test lung through the second opening to simulate the pleural cavity with a changeable compliance. The third opening was used to introduce the balloon into the glass chamber and was then sealed. Pressures were measured by different pressure transducers at three positions (Fig. 1): airway pressure at the proximal end of the endotracheal tube, directly measured chamber pressure to represent the surrounding pressure of the balloon, and balloon pressure.

During the bench procedure, mechanical ventilation was set as volume-controlled ventilation with constant flow of $60 \mathrm{l} / \mathrm{min}$; tidal volume of $500 \mathrm{ml}$; inspiratory time of $0.5 \mathrm{~s}$ with inspiratory pause time of $0.5 \mathrm{~s}$; respiratory rate of $20 \mathrm{breaths} / \mathrm{min}$; and positive end-expiratory pressure (PEEP) of $5 \mathrm{cmH}_{2} \mathrm{O}$. The inspiratory trigger was set at $-20 \mathrm{cmH}_{2} \mathrm{O}$ to avoid auto-triggering. The compliance of the Michigan test lung was set to $20 \mathrm{ml} / \mathrm{cmH}_{2} \mathrm{O}$. These ventilator and test lung settings produced a plateau airway pressure of approximately $40 \mathrm{cmH}_{2} \mathrm{O}$ and a chamber pressure of $20 \mathrm{cmH}_{2} \mathrm{O}$.

After the balloon was randomly introduced into the glass chamber described above, the model was tested by two methods to confirm that there was no systematic leak: (1) expiratory tidal volume no less than inspiratory tidal volume during mechanical ventilation and (2) decrease in airway pressure of no less than $1 \mathrm{cmH}_{2} \mathrm{O}$ during $30 \mathrm{~s}$ of end-expiratory occlusion at $30 \mathrm{cmH}_{2} \mathrm{O}$ of PEEP.

A three-way stopcock was connected to the balloon lumen of the catheter. Mechanical ventilation was not terminated during balloon manipulation to mimic an actual clinical scenario. The balloon was first deflated by generating a negative pressure, followed by opening the balloon to the atmosphere for $3 \mathrm{~min}$ to standardize the residual volume remaining in the balloon and the catheter lumen. The balloon was then progressively 
inflated in $0.5-\mathrm{ml}$ increments to a volume that resulted in a deviation in the balloon pressure from the chamber pressure that exceeded $1 \mathrm{cmH}_{2} \mathrm{O}$. Balloon inflation was performed at end-expiration. At each tested balloon volume, after 3-min equilibration period, endexpiratory and end-inspiratory occlusion was performed (each for $10 \mathrm{~s}$ ), and balloon pressure and chamber pressure were simultaneously measured during the last second.

\section{Balloon volume determination by sigmoid regression}

Balloon volume was plotted against balloon pressure, and these two variables were fitted to the following sigmoid regression equation [18-20]:

$$
\text { Balloon volume }=a+\frac{b}{1+e^{-(\text {Balloon pressure- }- \text { ) } / d}}
$$

The balloon pressure-volume curve was fitted using the Levenberg-Marquardt iterative algorithm, which was set to run until the change in the sum of squared residuals was lower than $10^{-8}$. An example scatter graph with fitted line is shown in Fig. $2 \mathrm{a}$. Equation 1 has four fitting parameters: $a$, in units of volume $(\mathrm{ml})$, representing the lower asymptote of the fitted sigmoid curve; $b$, also in units of volume $(\mathrm{ml})$, representing the vertical distance from the lower to the upper asymptote; $c\left(\mathrm{cmH}_{2} \mathrm{O}\right)$, representing the pressure at the midpoint of the sigmoid curve where the concavity changes direction; and $d\left(\mathrm{cmH}_{2} \mathrm{O}\right)$, which is proportional to the minimal balloon pressure change as the balloon volume increases. According to the characteristic features of the sigmoid curve, the maximal upward and downward curvature changes occurred at the lower and upper inflection points with balloon pressures equal to $c \pm 1.317 d$ (Fig. 2a) [21]. Within these two inflection points, the balloon pressure change was minimal during the progressively inflation of the balloon. We selected a narrower range for the estimation of optimal balloon volume and defined the balloon volume at balloon
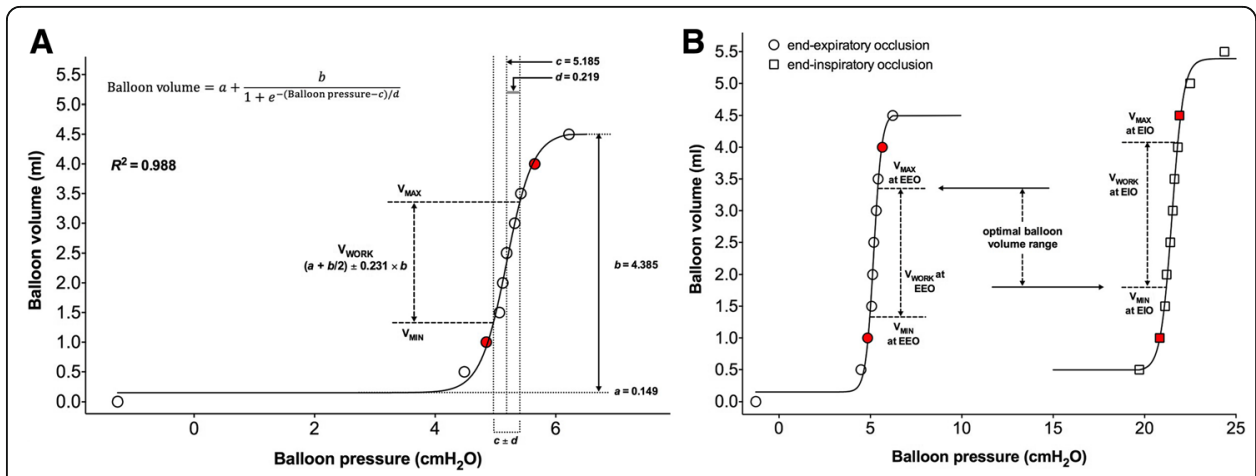

Fig. 2 An example of the fitted balloon volume-pressure curve using the sigmoid regression equation. a An example of the fitting of balloon pressure and balloon volume in a SmartCath-G balloon at end-expiratory occlusion during simulated mechanical ventilation. Circles are individual data points. The solid line represents the fitted curve. The equation (shown on the top of the figure) has four fitting parameters: $a$, in units of volume (ml), representing the lower asymptote of the fitted sigmoid curve; $b$, also in units of volume $(\mathrm{mll})$, representing the vertical distance from the lower to the upper asymptote; $\mathrm{C}\left(\mathrm{cmH}_{2} \mathrm{O}\right)$, representing the pressure at the midpoint of the sigmoid curve where the concavity changes direction; and $d\left(\mathrm{cmH}_{2} \mathrm{O}\right)$, is proportional to the minimal balloon pressure change as balloon volume increasing. We defined the balloon volume at balloon pressure of $c-d$ as the minimal inflating volume $\left(V_{\mathrm{MNN}}\right.$, equal to $\left.[a+b / 2]-0.231 b\right)$ and $c+d$ as the maximal volume $\left(V_{\text {MAx, }}\right.$ equal to $\left.[a+b / 2]+0.231 b\right)$ Within these two points, the balloon pressure change was minimal during the inflation of the balloon. Balloon working volume $\left(V_{\text {wORK }}\right)$ was defined as the difference between $V_{\text {MIN }}$ and $V_{\text {MAX }} \cdot \mathbf{b}$ The analysis of $V_{\text {work }} s$ during end-expiratory (EEO) and end-inspiratory occlusion (EIO) in the same balloon in $\mathbf{a}$. The $V_{\text {MIN }}$ and $V_{\text {MAX }}$ both satisfying the two phases were defined as the low and the high limit of the optimal balloon volume range 
pressure of $c-d$ as the minimal inflating volume $\left(V_{\mathrm{MIN}}\right.$, equal to $\left.[a+b / 2]-0.231 b\right)$ and $c+d$ as the maximal volume $\left(V_{\mathrm{MAX}}\right.$, equal to $\left.[a+b / 2]+0.231 b\right)$. The balloon $P_{\mathrm{TM}}$ values at $V_{\mathrm{MIN}}$ and $V_{\mathrm{MAX}}$ were calculated as the estimated balloon pressure minus the averaged chamber pressure. Acceptable accuracy of measurement was defined as a $P_{\text {TM }}$ within $\pm 1 \mathrm{cmH}_{2} \mathrm{O}$ [15]. Balloon working volume ( $\left.V_{\text {WORK }}\right)$ was calculated as the difference between $V_{\mathrm{MIN}}$ and $V_{\mathrm{MAX}}$. Under atmospheric condition, balloon elastance was estimated as the difference in balloon pressure between $V_{\mathrm{MIN}}$ and $V_{\mathrm{MAX}}$ divided by $V_{\text {WORK }}$.

In each tested balloon, $V_{\text {WORK }}$ values during end-expiratory and end-inspiratory occlusion were explored, and the $V_{\mathrm{MIN}}$ and $V_{\mathrm{MAX}}$ providing accurate measurement at both phases were defined as the low and the high limits of the optimal balloon volume range (Fig. 2b). Visual inspection of the intermediate linear section of the balloon pressure-volume curve was also performed using the method reported by Mojoli et al. [17]. Balloon $P_{\mathrm{TM}}$ values at $V_{\mathrm{MIN}}$ and $V_{\mathrm{MAX}}$ obtained using the visual inspection method were also calculated.

\section{Statistical analysis}

In the balloon pressure-volume curve analysis, the best-fit coefficient $R^{2}$ and the bias-corrected Akaike information criterion (AICc) of the sigmoid regression were calculated [22]. To assess the overall goodness-of-fit in the regression equation of each type of balloon using each corresponding fitted equation, the balloon volume and balloon pressure were transformed to dimensionless variables (balloon volume $-a$ ) $/ b$ and (balloon pressure $-c) / d$, which allowed these variables to be plotted on the same horizontal and vertical axes. These two dimensionless variables were fitted to the following equation $[19,20]$ :

(balloon volume $-a) / b=\frac{1}{1+e^{-(\text {balloon pressure-c)/d }}}$

The mean and standard deviation (SD) of the residual for (balloon volume $-a$ ) $/ b$ were calculated and plotted against (balloon pressure $-c$ )/d.

Categorical variables were expressed as counts and percentages. Continuous data were checked for normal distribution using the Kolmogorov-Smirnov test and were presented as mean \pm SD or as median (25th to 75th percentile) as appropriate. Balloon volume data at atmospheric pressure were compared across different balloon types using one-way ANOVA and the Student-Newman-Keuls test for pairwise comparisons. Under dynamic conditions during simulated passive ventilation, one-way ANOVA was also used to compare chamber pressure and balloon pressure at different balloon volume. Repeated measures of analysis of variance was used to compare balloon volume data across different balloon types and between end-expiratory and end-inspiratory occlusion. The paired $t$ test was used to compare the difference in the absolute $P_{\mathrm{TM}}$ at $V_{\mathrm{MIN}}$ or $V_{\text {MAX }}$ between the sigmoid fitting and visual inspecting methods.

All tests of significance were at the 5\% significance level. Analyses were conducted using SPSS V.20.0 (SPSS Inc., Chicago, IL, USA).

\section{Results}

Sigmoid fitting parameters for all tested balloons and under all bench conditions are shown in Additional file 1: Table S1. When data obtained under all tested conditions 
for each type of balloon were pooled and normalized according to the parameters in each corresponding regression equation (Eq. 1), the dimensionless variable (balloon volume $-a) / b$ was fitted closely with (balloon pressure $-c$ ) $/ d$ along the standard sigmoid curve (Additional file 1: Figure S1). The mean \pm SD of residual of (balloon volume $-a) / b$ was $0.000047 \pm 0.0358,0.00000 \pm 0.0554$, and $0.000028 \pm 0.0648$ for the Cooper, SmartCath-G, and Microtek catheters, respectively. Residuals of (balloon volume $-a) / b$ were scattered evenly against (balloon pressure $-c) / d$ for the Cooper catheter, but a statistically positive correlation was found for the SmartCath-G and the Microtek catheters (Additional file 1: Figure S2).

Table 1 shows the characteristics of the tested balloons. The geometric volume of the balloon was $2.8 \mathrm{ml}$ for the Cooper, $5.3 \mathrm{ml}$ for the SmartCath-G, and $7.5 \mathrm{ml}$ for the Microtek catheter. At atmospheric pressure, there were significant differences in $V_{\mathrm{MIN}}, V_{\mathrm{MAX}}$, $V_{\text {WORK}}$, and balloon elastance across the different balloon types $(p<0.001$, Fig. 3$)$.

There was no systematic leak in the dynamic model during simulated passive ventilation. With progressive increments of balloon injection volume, the chamber pressure remained stable, but balloon pressure increased significantly in each type of catheter at end-expiratory or end-inspiratory occlusion (all $p<0.001$ ) (Additional file 2: Figure S3). Balloon volume data during dynamic ventilation are shown in Fig. 4. There were significant differences in $V_{\mathrm{MIN}}, V_{\mathrm{MAX}}$, and $V_{\mathrm{WORK}}$ across different balloon types and between end-expiratory and end-inspiratory occlusion $(p=0.003$ to $<0.001)$. During either end-expiratory or end-inspiratory occlusion, $V_{\mathrm{MIN}}, V_{\mathrm{MAX}}$, and $V_{\text {WORK }}$ were in a significant ascending order of Cooper $<$ SmartCath-G $<$ Microtek catheters, in accordance with the sequence of geometric volume. The three balloon volume parameters during end-inspiratory occlusion were significantly larger than those during endexpiratory occlusion, except for $V_{\mathrm{MAX}}$ in the Cooper $(p=0.062)$ and $V_{\text {WORK }}$ in the Microtek ( $p=0.363)$ (Fig. 4). In all tested balloons, all $P_{\mathrm{TM}}$ values under the different tested conditions (atmospheric pressure and simulated ventilation) were within $\pm 1.0 \mathrm{cmH}_{2} \mathrm{O}$ (Fig. 5). All $V_{\text {MIN }}$ and $V_{\text {MAX }}$ determined by the sigmoid regression were within the intermediate linear section obtained by visually inspecting the balloon pressure-volume curve (Additional file 3: Table S2). For either $V_{\text {MIN }}$ or $V_{\text {MAX }}$, absolute $P_{\mathrm{TM}}$ values as measured by the sigmoid fitting were lower than those measured by the intermediate linear section inspection (Additional file 3: Figure S4).

For each tested balloon, an optimal balloon volume range existed that provided accurate measurement during both end-expiratory and end-inspiratory occlusion, which was $0.7 \pm 0.0$ to $1.7 \pm 0.2 \mathrm{ml}$ for the Cooper, $1.9 \pm 0.2$ to $3.6 \pm 0.3 \mathrm{ml}$ for the SmartCath-G, and $2.2 \pm 0.2$ to $4.6 \pm 0.1 \mathrm{ml}$ for the Microtek catheter (Fig. 6).

Table 1 Balloon material and dimension characteristics

\begin{tabular}{llll}
\hline Balloon material & Cooper & SmartCath-G & Microtek \\
\hline Length of the balloon $(\mathrm{mm})$ & Polyethylene & Polyethylene & Latex \\
Diameter of the balloon $(\mathrm{mm})$ & 92.5 & 109.3 & 105.2 \\
Geometric volume of the balloon ${ }^{\mathrm{a}}(\mathrm{ml})$ & 6.3 & 10.4 & 9.7 \\
Recommended inflating volume $(\mathrm{ml})$ & 2.8 & 5.3 & 7.5 \\
\hline
\end{tabular}

${ }^{a}$ Geometric volume of the balloon was calculated by the length and diameter of the balloon, using the capsule body as approximation. Because the feeding tube passed through the inner space of the balloon in SmartCath- $G$ catheter, this volume was deducted in the calculation of balloon geometric volume in this type of catheter 

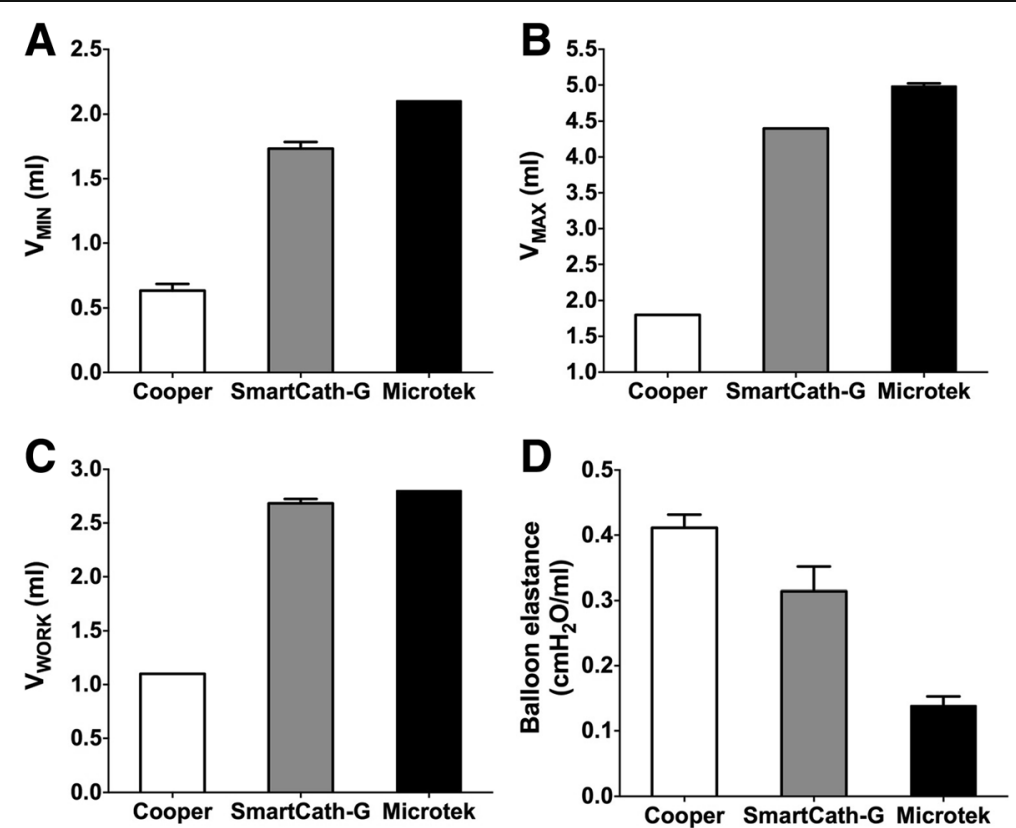

Fig. 3 a-d Balloon volume data and balloon elastance at atmosphere. Data are shown as mean and standard deviation. Significant difference existed in minimal $\left(V_{\text {MIN }}, \mathbf{a}\right)$, maximal balloon inflating volume $\left(V_{\text {MAX }}, \mathbf{b}\right)$, working volume $\left(V_{\text {WORK, }} \mathbf{C}\right)$, and balloon elastance across different balloons $(p<0.001, \mathbf{d})$. All $p$ values are $<0.001$ in pairwise comparison

\section{Discussion}

In the present study, we used a mathematical method, sigmoid regression, to analyze the balloon pressure-volume curve and to determine the optimal balloon volume under different bench conditions. Our main findings are:

(1) An optimal balloon volume range exists that provides accurate measurement at both end-expiration and end-inspiration for each of the tested balloon.

(2) The sigmoid regression fits the balloon pressure-volume curve excellently and enables an accurate estimation of the optimal balloon volume. This method provides an additional way to determine the optimal balloon volume.

Proper titration of balloon volume is critical for the accurate measurement of esophageal pressure $[1,2]$. In bench studies, the optimal balloon volume was defined as a volume range in which $P_{\mathrm{TM}}$ was within a certain threshold $[14,15]$. In the present study,

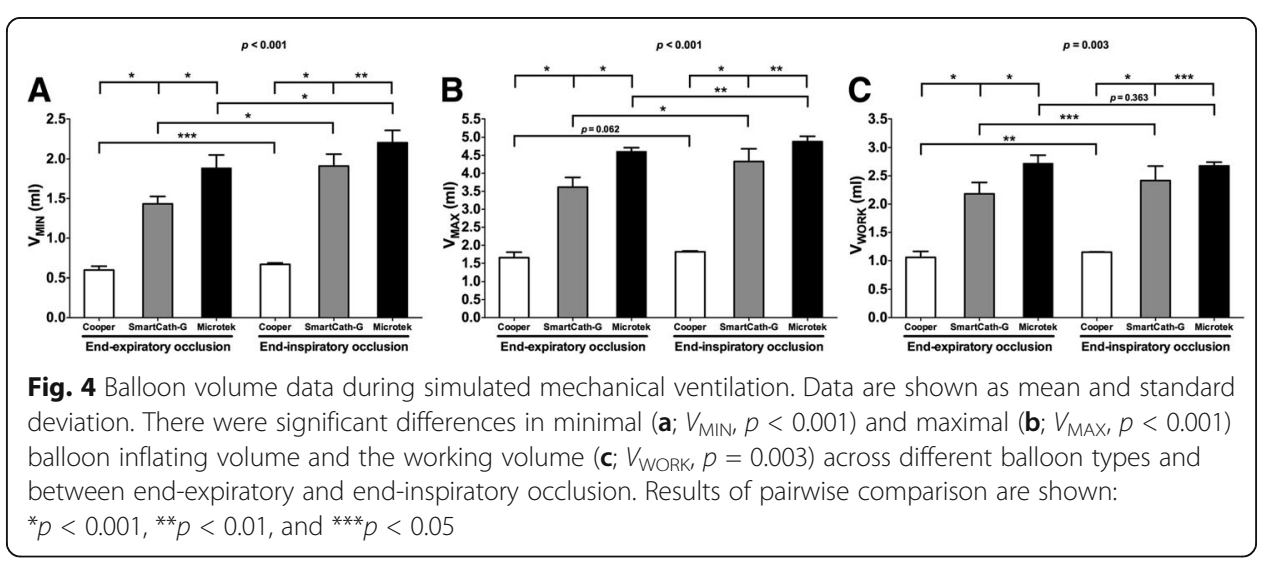




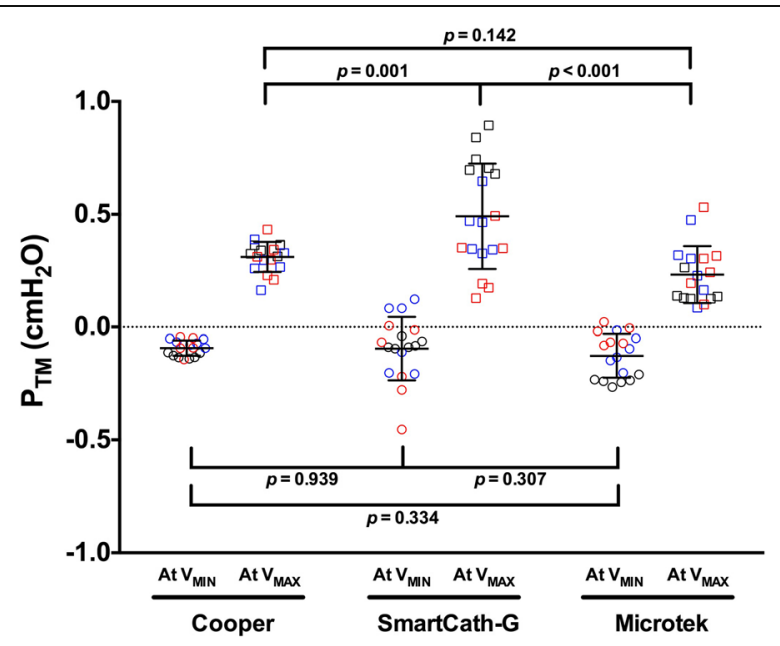

Fig. 5 Balloon transmural pressure under all bench conditions. Balloon transmural pressure $\left(P_{T M}\right)$ at minimal $\left(V_{\text {MIN }}\right)$ and maximal balloon inflating volume $\left(V_{\text {MAX }}\right)$ under atmosphere (black) and end-expiratory (blue) and end-inspiratory occlusion (red) during simulated passive ventilation. All $P_{\mathrm{TM}}$ were within $\pm 1 \mathrm{CmH}_{2} \mathrm{O}$. Mean and standard deviation are also shown

we estimated the optimal balloon volume and range using a sigmoid fitting of balloon pressure-volume curve, which has been used to predict the lower and upper inflection points in the respiratory system pressure-volume curve analysis [19, 20]. After normalization based on parameters of the regression equation, the dimensionless variables of balloon pressure and balloon volume were closely distributed along the standard sigmoid curve, and the mean residual for the dimensionless volume variables was minor (Additional file 1: Figure S1 and S2). More importantly, all measured $P_{\mathrm{TM}}$ values at $V_{\text {MIN }}$ and $V_{\text {MAX }}$ were within $\pm 1 \mathrm{cmH}_{2} \mathrm{O}$ (Fig. 5), which has been used as the threshold in previous bench study [15]. Together, these results indicate that the sigmoid regression of balloon pressure and volume can be used to accurately estimate the optimal filling volume.

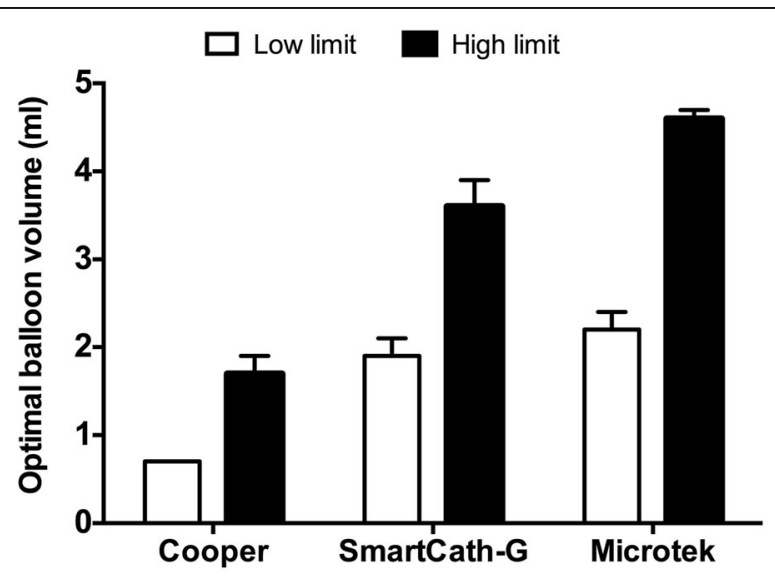

Fig. 6 The low and high limit of optimal balloon volumes that provide accurate measurement during both end-expiratory and end-inspiratory occlusion. The low and high limits were defined as the minimal and maximal balloon inflating volume that provided accurate measurement during both end-expiratory and end-inspiratory occlusion 
In the present study, in addition to testing the balloons under atmospheric condition, we also analyzed the balloon pressure-volume relationship during simulated passive ventilation in a respiratory system model (Fig. 1), which has been reported for educational purposes $[23,24]$. We employed this model in an attempt to mimic actual clinical scenarios. Using our sigmoid fitting method, we confirmed previous two major findings regarding the relationship between balloon filling volume and geometric volume and surrounding pressure $[14,15]$. First, the balloon filling volume is determined by the geometric volume of the balloon (Fig. 3): the larger the geometric volume, the larger the balloon filling volume that is needed to reflect surrounding pressure. Second, the balloon filling volume required for accurate measurement increases as the balloon surrounding pressure increases (Fig. 4). The latter finding is of clinical significance during esophageal monitoring in passive patients under controlled ventilation. Because pleural pressure increases during inspiration, the balloon volume suitable for measuring during the end-expiratory phase may not be adequate for measurement during the end-inspiratory phase. Fortunately, this did not occur in our bench model with simulated pleural pressures ranging from $5 \mathrm{cmH}_{2} \mathrm{O}$ during end-expiratory occlusion to $20 \mathrm{cmH}_{2} \mathrm{O}$ during end-inspiratory occlusion. An optimal volume range existed that enabled accurate measurement at both phases (Fig. 6).

The slope of the intermediate section is minimal under in vitro conditions and is related to balloon elastance $[15,16]$, but is usually inclined in vivo due to esophageal elastance [17]. We estimated the balloon elastance based on the fitted balloon pressure and volume data, and our results $\left(0.1\right.$ to $0.4 \mathrm{cmH}_{2} \mathrm{O} / \mathrm{ml}$, Fig. $\left.3 \mathrm{~d}\right)$ were comparable to those reported by Cross et al. [16]. Further investigation is warranted to determine whether this method can improve the accuracy of esophageal elastance measurement and esophageal pressure calibration at the bedside.

In clinical settings, because it is difficult to obtain the $P_{\mathrm{TM}}$, the proper balloon volume is usually determined by visual inspecting the intermediate linear section of the balloon pressure-volume curve [17]. Compared to the visual inspection method, fitting the data to a sigmoid curve yielded $V_{\text {WORK }}$ with a narrower range and $P_{\mathrm{TM}}$ closer to zero (Additional file 3: Table S2 and Figure S4). These results suggested that our introduced method might provide a stricter estimation of the optimal balloon volume. Additionally, sigmoid fitting is objective and based on mathematics. However, because the sigmoid fitting method was only investigated in a bench model in the present study, further study is needed to determine whether this method can improve the assessment of optimal balloon volume. Since a relatively low adequate balloon volume is recommended to avoid overfilling [2,11], we suggest using the low limit of the optimal balloon volume range (Fig. 6), thus avoiding overfilling of the balloon during expiration and simultaneously avoiding underfilling during inspiration.

There are limitations to the present study. First, in our bench model, we only simulated the pleural cavity without the esophagus. It has been shown that esophageal wall elastance significantly influences esophageal pressure monitoring [17]. Therefore, our findings cannot be directly translated into clinical practice. Moreover, the procedure was only performed during controlled ventilation, and the findings cannot be applied to patients with spontaneous breathing. Second, the high balloon surrounding pressure simulated in the present study was somewhat lower than the end-inspiratory esophageal pressure in patients with acute respiratory distress syndrome during passive 
ventilation [5-7]. The titration of balloon volume needs further investigation in clinical studies, especially in patients with relatively high esophageal pressure and airway driving pressure.

\section{Conclusions}

The balloon pressure-volume curve is fitted well by sigmoid regression and provides an accurate estimation of the balloon volume. Careful titration of the filling volume should be performed in clinical practice because the adequate balloon volume is discrepant among different types of balloon at different surrounding pressures. In each of the tested balloon, an optimal filling volume range exists that provides accurate measurement during both end-expiratory and end-inspiratory occlusion.

\section{Additional files}

Additional file 1: Table S1. Sigmoid fitting parameters at atmospheric pressure and end-expiratory and end-inspiratory occlusion. Figure S1. Fitting of the dimensionless variables in balloon pressure-volume sigmoid regression equation. Figure S2. Plot of residual of (balloon volume $-a) / b$ predicted by a standard sigmoid fitting against (balloon pressure - c)/d. (PDF $599 \mathrm{~kb}$ )

Additional file 2: Figure S3. Chamber pressure and balloon pressure during balloon volume manipulation at atmospheric pressure and under simulated passive ventilation. (PDF $212 \mathrm{~kb}$ )

Additional file 3: Table S2. Minimal and maximal balloon inflating volume estimated by sigmoid fitting and by visual inspection of the intermediate linear section of the balloon pressure-volume curve during end-expiratory and end-inspiratory occlusion. Figure S4. Absolute balloon transmural pressure at minimal and maximal balloon inflating volume. (PDF 329 kb)

\section{Abbreviations}

PEEP: Positive end-expiratory pressure; $P_{\mathrm{TM}}$ : Balloon transmural pressure; SD: Standard deviation; $V_{\mathrm{MAX}}$ : Maximal balloon inflating volume; $V_{\text {MIN }}$ : Minimal balloon inflating volume; $V_{\text {work: }}$ Balloon working volume

\section{Acknowledgements}

We would like to thank Prof. Yi-Long Wang (Clinical Trial and Research Center, Beijing Tiantan Hospital, Capital Medical University) for his valuable suggestions on the statistical analysis.

\section{Funding}

This study was supported by grants from the Beijing Municipal Administration of Hospital (DFL20150502) and Special Program of Beijing Municipal Science \& Technology Commission (Z161100000116081). The sponsor had no role in the study design, data collection, data analysis, data interpretation, or writing of the report.

\section{Availability of data and materials}

Detailed data supporting the findings are available at Dataset on ResearchGate (https://www.researchgate.net/publication/ 310415196_Use_of_sigmoid_fitting_to_determine_the_optimal_balloon_volume_of_esophageal_balloon_catheter_an_ in_vitro_bench_experiment?ev=prf_pub).

\section{Authors' contributions}

$Y L Y, X H, M X$, and JXZ designed the study design. $Y L Y, X H, M X$, and $H C$ performed the bench procedure and collected the experimental data. YLY, XH, ZHS, and GQC analyzed the data. XMS, XYL, KS, and JXZ wrote the paper. JXZ reviewed the paper and approved the final manuscript. All of the authors read and approved the final manuscript.

Ethics approval and consent to participate

Not applicable.

Consent for publication

Not applicable.

Competing interests

The authors declare that they have no competing interests.

\section{Publisher's Note}

Springer Nature remains neutral with regard to jurisdictional claims in published maps and institutional affiliations. 


\section{Author details}

'Department of Critical Care Medicine, Beijing Tiantan Hospital, Capital Medical University, No 6, Tiantan Xili, Dongcheng District, Beijing 100050, China. Intensive Care Unit, Beijing Electric Power Hospital, Capital Medical University, Beijing 100073, China. ${ }^{3}$ Surgical Intensive Care Unit, Fujian Provincial Clinical College Hospital, Fujian Medical University, Fuzhou 350001, China.

Received: 19 November 2016 Accepted: 18 July 2017

Published online: 02 August 2017

\section{References}

1. Akoumianaki E, Maggiore SM, Valenza F, Bellani G, Jubran A et al (2014) The application of esophageal pressure measurement in patients with respiratory failure. Am J Respir Crit Care Med 189:520-531

2. Mauri T, Yoshida T, Bellani G, Goligher EC, Carteaux G et al (2016) Esophageal and transpulmonary pressure in the clinical setting: meaning, usefulness and perspectives. Intensive Care Med 42:1360-1373

3. Jubran A, Grant BJ, Laghi F, Parthasarathy S, Tobin MJ (2005) Weaning prediction: esophageal pressure monitoring complements readiness testing. Am J Respir Crit Care Med 171:1252-1259

4. Kallet RH, Hemphill JC 3rd, Dicker RA, Alonso JA, Campbell AR et al (2007) The spontaneous breathing pattern and work of breathing of patients with acute respiratory distress syndrome and acute lung injury. Respir Care 52: 989-995

5. Talmor D, Sarge T, O'Donnell CR, Ritz R, Malhotra A et al (2006) Esophageal and transpulmonary pressures in acute respiratory failure. Crit Care Med 34:1389-1394

6. Talmor D, Sarge T, Malhotra A, O'Donnell CR, Ritz R et al (2008) Mechanical ventilation guided by esophageal pressure in acute lung injury. N Engl J Med 359:2095-2104

7. Chiumello D, Carlesso E, Brioni M, Cressoni M (2016) Airway driving pressure and lung stress in ARDS patients. Crit Care 20:276

8. Persson $\mathrm{P}$, Lundin S, Stenqvist $\mathrm{O}$ (2016) Transpulmonary and pleural pressure in a respiratory system model with an elastic recoiling lung and an expanding chest wall. Intensive Care Med Exp 4:26

9. Mead J, Mcllroy MB, Selverstone NJ, Kriete BC (1955) Measurement of intraesophageal pressure. J Appl Physiol 7: 491-495

10. Petit JM, Milic-Emili G (1958) Measurement of endoesophageal pressure. J Appl Physiol 13:481-485

11. Milic-Emili J, Mead J, Turner JM, Glauser EM (1964) Improved technique for estimating pleural pressure from esophageal balloons. J Appl Physiol 19:207-211

12. Lemen R, Benson M, Jones JG (1974) Absolute pressure measurements with hand-dipped and manufactured esophageal balloons. J Appl Physiol 37:600-603

13. Beardsmore CS, Helms P, Stocks J, Hatch DJ, Silverman M (1980) Improved esophageal balloon technique for use in infants. J Appl Physiol Respir Environ Exerc Physiol 49:735-742

14. Walterspacher S, Isaak L, Guttmann J, Kabitz HJ, Schumann S (2014) Assessing respiratory function depends on mechanical characteristics of balloon catheters. Respir Care 59:1345-1352

15. Mojoli F, Chiumello D, Pozzi M, Algieri I, Bianzina S et al (2015) Esophageal pressure measurements under different conditions of intrathoracic pressure. An in vitro study of second generation balloon catheters. Minerva Anestesiol 81:855-864

16. Cross TJ, Lalande S, Hyatt RE, Johnson BD (2015) Response characteristics of esophageal balloon catheters handmade using latex and nonlatex materials. Physiol Rep 3:e12426

17. Mojoli F, lotti GA, Torriglia F, Pozzi M, Volta CA et al (2016) In vivo calibration of esophageal pressure in the mechanically ventilated patient makes measurements reliable. Crit Care 20:98

18. Smyth GK (2002) Nonlinear regression. In: El-Shaarawi AH, Piegorsch WW (eds) Encyclopedia of environmetrics. John Wiley \& Sons, Chichester

19. Venegas JG, Harris RS, Simon BA (1998) A comprehensive equation for the pulmonary pressure-volume curve. J Appl Physiol 84:389-395

20. Harris RS, Hess DR, Venegas JG (2000) An objective analysis of the pressure-volume curve in the acute respiratory distress syndrome. Am J Respir Crit Care Med 161:432-439

21. Narusawa U (2001) General characteristics of the sigmoidal model equation representing quasi-static pulmonary P-V curves. J Appl Physiol 91:201-210

22. Spiess AN, Neumeyer N (2010) An evaluation of R2 as an inadequate measure for nonlinear models in pharmacological and biochemical research: a Monte Carlo approach. BMC Pharmacol 10:6

23. Chinet AE (1989) Chest-lung statics: a realistic analog for student laboratory. Am J Phys 257:S9-10

24. Anderson J, Goplen C, Murray L, Seashore K, Soundarrajan M (2009) Human respiratory mechanics demonstration model. Adv Physiol Educ 33:53-59 